\title{
Consensus Needed for Noncontrast CT Markers in Intracerebral Hemorrhage
}

W e read with interest the article by $\mathrm{Ng}$ et al, in which the authors investigated associations between the "swirl sign" and hematoma growth. $\mathrm{Ng}$ et al found that patients positive for the swirl sign demonstrated larger hemorrhage volumes and higher odds of subsequent hemorrhage expansion (HE). ${ }^{1}$

As the authors mention, accurately predicting HE is key for targeting those patients at higher risk of this ominous outcome, hence deemed to be most likely to benefit from expansion-targeted treatment such as intensive blood pressure lowering or hemostatic treatment. Consequently, acute imaging biomarkers associated with HE have been the subject of intense research effort. In this context, the article by $\mathrm{Ng}$ et al would have thus benefited from a more complete and comprehensive account of the relevant literature in the field. As it stands, references beyond the spot sign are only seldom, if at all, cited in the current article.

Indeed, the first convincing evidence of a relationship between hemorrhage heterogeneity and intracerebral hemorrhage (ICH) outcome came as early as $2008 .^{2}$ After that, a number of studies investigated several aspects of acute intracerebral hemorrhage using noncontrast CT to look for HE and outcome, coining a new term for each of those particular aspects ("black hole sign," "island sign," "satellite sign," "blend sign," "density heterogeneity scale," and so forth). ${ }^{2}$ One downside of giving a different name to each individual sign in the literature is that it may gray out their potential translation in clinical practice by a profusion of potentially overlapping sets of findings. For instance, because the black hole sign represents a subcategory of the swirl sign and because an important degree of heterogeneity should characterize the presence of all "swirls" and "black holes," the relative value of each of these findings is very hard to evaluate pragmatically. ${ }^{2}$

There is little remaining doubt that ICH heterogeneity based on noncontrast CT is associated with more frequent hemorrhagic expansion and poorer clinical outcome. However, there is considerable overlap between different noncontrast CT predictors of $\mathrm{HE}$, with lack of consensus on the diagnostic criteria to identify these markers. Hence, future research should aim to homogenize the ever-growing terminology of signs predicting HE, analyzing whether they ought to be used for patient selection in ICH trials

http://dx.doi.org/10.3174/ajnr.A5604 and validation studies. Because one of the common features of these markers is the presence of a hypodense area within the hyperdense acute hematoma, their simplest descriptive characterization (eg, "presence of hypodense area encapsulated in the hematoma, with no contact with the hemorrhage margin") is the most replicable and straightforward way of assessing hemorrhage heterogeneity. In fact, in a cohort of 1029 patients, we found that the presence of any hypodensity was strongly associated with ICH expansion $^{3}$ and outcome ${ }^{4}$ and that dividing these hypodensities into their various aspects (corresponding to the above-mentioned markers, including the swirl sign investigated by $\mathrm{Ng}$ et al) did not change their predictive ability for $\mathrm{HE}$, independent of power issues. ${ }^{3}$

In a large post hoc analysis of patients enrolled in the Antihypertensive Treatment of Acute Cerebral Hemorrhage II (ATACH-II) randomized controlled trial, there was no evidence that patients with these markers, hypothesized to be at the highest risk of expansion, specifically benefited from intensive blood pressure reduction aimed at limiting hemorrhage growth. ${ }^{5}$ These and other findings reinforce the notion that these individual markers may not be sufficient, sensitive, or calibrated enough to identify target patients for such therapies with high sensitivity and specificity.

While there is hope of finding better acute therapeutic approaches for patients with $\mathrm{ICH}$, harmonizing nomenclature terminology, scales, and assessment methods is the way forward.

\section{REFERENCES}

1. $\mathrm{Ng} \mathrm{D}$, Churilov L, Mitchell $\mathrm{P}$, et al. The CT swirl sign is associated with hematoma expansion in intracerebral hemorrhage. AJNR Am J Neuroradiol 2018;39:232-37 CrossRef Medline

2. Boulouis G, Morotti A, Charidimou A, et al. Noncontrast computed tomography markers of intracerebral hemorrhage expansion. Stroke 2017;48:1120-25 CrossRef Medline

3. Boulouis G, Morotti A, Brouwers HB, et al. Association between hypodensities detected by computed tomography and hematoma expansion in patients with intracerebral hemorrhage. JAMA Neurol 2016;73:961-68 CrossRef Medline

4. Boulouis G, Morotti A, Brouwers HB, et al. Noncontrast computed tomography hypodensities predict poor outcome in intracerebral hemorrhage patients. Stroke 2016;47:2511-16 CrossRef Medline

5. Morotti A, Boulouis G, Romero JM, et al; ATACH-II and NETT investigators. Blood pressure reduction and noncontrast CT markers of 
intracerebral hemorrhage expansion. Neurology 2017;89:548-54 CrossRef Medline

(D) G. Boulouis Department of Neuroradiology Institut National de la Santé et de la Recherche Médicale Unite Mixte de Recherche 894 Centre Hospitalier Sainte-Anne, Université Paris-Descartes Paris, France Department of Neurology, Hemorrhagic Stroke Research Program Massachusetts General Hospital Stroke Research Center, Harvard Medical School Boston, Massachusetts

(D) A. Charidimou

Department of Neurology, Hemorrhagic Stroke Research Program Massachusetts General Hospital Stroke Research Center, Harvard Medical School Boston, Massachusetts

DA. Morotti Stroke Unit Istituto Di Ricovero e Cura a Carattere Scientifico Mondino Foundation Pavia, Italy 